\title{
O cuidado farmacêutico na melhora da adesão ao tratamento medicamentoso
}

\author{
Pharmaceutical care in improving adherence to drug treatment \\ Atención farmacéutica para mejorar la adhesión al tratamiento con drogas
}

Recebido: 22/11/2021 | Revisado: 02/12/2021 | Aceito: 03/12/2021 | Publicado: 12/12/2021

\author{
Jamilly Fernanda Brito Rodrigues \\ ORCID: https://orcid.org/0000-0002-6355-7397 \\ Universidade Federal de Pernambuco, Brasil \\ E-mail: jamillyfernanda_@hotmail.com \\ Dayzyane Farias dos Santos Melo \\ ORCID: https://orcid.org/0000-0001-6095-5691 \\ Universidade Federal de Pernambuco, Brasil \\ E-mail: dayzyane.farias@gmail.com \\ Wylma Danuzza Guimarães Bastos \\ ORCID: https://orcid.org/0000-0002-9908-4237 \\ Universidade Federal de Pernambuco, Brasil \\ E-mail: wylmabastos@yahoo.com.br \\ Karina Perrelli Randau \\ ORCID: https://orcid.org/0000-0002-4486-4420 \\ Universidade Federal de Pernambuco, Brasil \\ E-mail:krandau@hotmail.com
}

\begin{abstract}
Resumo
A adesão ao tratamento medicamentoso depende de um conjunto de fatores associados, e é caracterizada como crucial para a execução adequada do tratamento. A má adesão pode ser um agravante para as condições de saúde e proporcionar um aumento da mortalidade. Uma das atividades que compõe o cuidado farmacêutico é a promoção e a manutenção da adesão ao tratamento. Portanto, esse trabalho tem como objetivo evidenciar a importância do cuidado farmacêutico e do farmacêutico no processo de adesão. Foi realizada uma revisão literária, a partir da pesquisa nos bancos de dados PubMed, Google Scholar e Literatura Latino-Americana e do Caribe em Ciências da Saúde, com o recorte temporal de 2010 a 2021. Após realização da triagem e aplicação dos critérios de inclusão e exclusão, foram selecionados 16 trabalhos. A partir destes, foi evidenciado que o cuidado farmacêutico foi aplicado a diferentes condições de saúde, a partir de diferentes tipos de intervenções farmacêuticas. Estudos demonstraram melhora na adesão ao tratamento medicamentoso quando associado ao cuidado farmacêutico, em diferentes contextos da saúde, e a partir de uma pluralidade de tipos de intervenções. Portanto, é possível concluir que o cuidado farmacêutico se mostra como uma prática estratégica e de extrema importância para promoção da adesão medicamentosa.
\end{abstract}

Palavras-chave: Adesão à medicação; Cuidados farmacêuticos; Farmácia.

\begin{abstract}
Adherence to drug treatment depends on a set of associated factors, and is characterized as crucial for the proper execution of the treatment. Poor adherence can aggravate health conditions and lead to an increase in mortality. One of the activities that make up pharmaceutical care is the promotion and maintenance of treatment adherence. Therefore, this work aims to highlight the importance of the pharmacist and pharmacist care in the adherence process,. A literature review was carried out, based on a search in the PubMed, Google Scholar and Latin American and Caribbean Literature in Health Sciences databases, with a time frame from 2010 to 2021. After screening and applying the inclusion and exclusion criteria, 16 papers were selected.. From these, it was evidenced that pharmaceutical care was applied to different health conditions, from different types of pharmaceutical interventions. Studies have shown improved adherence to drug treatment when associated with pharmaceutical care, in different health contexts, and from a variety of types of interventions. Therefore, it is possible to conclude that pharmaceutical care is shown as a strategic and extremely important practice to promote medication adherence.
\end{abstract}

Keywords: Medication adherence; Pharmaceutical services; Pharmacy.

\section{Resumen}

La adherencia al tratamiento farmacológico depende de un conjunto de factores asociados y se caracteriza por ser crucial para la correcta ejecución del tratamiento. La mala adherencia puede agravar las condiciones de salud y provocar un aumento de la mortalidad. Una de las actividades que integran la atención farmacéutica es la promoción y mantenimiento de la adherencia al tratamiento. Por tanto, este trabajo tiene como objetivo resaltar la importancia de la atencíon del farmacêutico y farmacéutico en el proceso de adherencia. Se realizó una revisión de la literatura, a partir de una búsqueda en las bases de datos PubMed, Google Scholar y Literatura Latinoamericana y del Caribe en 
Ciencias de la Salud, con un marco temporal de 2010 a 2021. Después de seleccionar y aplicar los criterios de inclusión y exclusión, se seleccionaron 16 artículos. A partir de estos, se evidenció que la atención farmacéutica se aplicó a diferentes condiciones de salud, desde diferentes tipos de intervenciones farmacéuticas. Los estudios han demostrado una mejor adherencia al tratamiento farmacológico cuando se asocia con la atención farmacéutica, en diferentes contextos de salud y a partir de una variedad de tipos de intervenciones. Por tanto, es posible concluir que la atención farmacéutica se muestra como una práctica estratégica y de suma importancia para promover la adherencia a la medicación.

Palabras clave: Cumplimiento de la medicación; Servicios farmacéuticos; Farmacia.

\section{Introdução}

Ao longo do tempo, o papel do farmacêutico sofreu uma série de transformações que resultaram em uma forma de atuação, e em um saber autocentrado no medicamento (Nicoletti \& Ito, 2018; Saturnino et al., 2012). A proposta da educação, aplicada e voltada para formação farmacêutica, contribuiu para tal delineamento, a partir da adoção de um modelo mais tecnicista (Nicoletti \& Ito, 2018).

Em contrapartida, o panorama atual revela uma mudança no perfil do farmacêutico, que passa a ter o cuidado ao paciente como foco e não mais, o medicamento em si (Nicoletti \& Ito, 2018). Esse novo paradigma surge com o advento da farmácia clínica e da atenção farmacêutica, que reorientam a prática farmacêutica para o âmbito social, com a atenção voltada para o paciente, a fim de garantir a qualidade de vida (Nicoletti \& Ito, 2018; Soares et al., 2016).

A atenção farmacêutica ou cuidado farmacêutico pode ser definido como um modelo de prática profissional que consiste na prestação de serviços que visam a promoção, prevenção e recuperação da saúde, e que tem como objetivo alcançar metas terapêuticas estabelecidas, em busca da qualidade de vida (Agostini, 2018; Cruz et al., 2020; Saturnino et al., 2012).

Dentre os serviços que compõe o cuidado farmacêutico, está a avaliação e promoção da adesão ao tratamento (Araujo et al., 2017). A adesão corresponde ao grau em que o comportamento de uma pessoa está de acordo com as recomendações de um profissional de saúde (Araujo et al., 2017; Organização Mundial da Saúde [OMS], 2003). E a evolução clínica positiva e o sucesso do tratamento estão diretamente relacionados a adesão (Menditto, 2020).

O cuidado farmacêutico representa para os pacientes, uma perspectiva de melhoria na qualidade de vida, a partir de uma melhora na adesão, visto que o farmacêutico atua no cuidado direto ao paciente, o que possibilita a sensibilização e orientação do paciente e seus familiares quanto à patologia, informações e peculiaridades sobre o tratamento, seus benefícios, e como realizá-lo, além de permitir a identificação de barreiras que dificultam a adesão (Agostini, 2018; Araujo, 2017; Bastosbarbosa, 2012).

Portanto, este trabalho teve como objetivo evidenciar, a partir de uma revisão literária, a importância do cuidado farmacêutico no processo de adesão ao tratamento medicamentoso, explorando as condições clínicas às quais foi aplicado e os tipos de intervenções realizadas pelo farmacêutico.

\section{Metodologia}

O estudo consiste em uma revisão literária. Foram utilizadas como bases de dados para a realização da pesquisa, o PubMed, o Google Scholar e a Literatura Latino-Americana e do Caribe em Ciências da Saúde (LILACS). O recorte temporal empregado foi de 2010 a maio de 2021. A busca ocorreu a partir da utilização dos seguintes descritores, vinculados pelo operador booleano AND: pharmaceutical care, adherence, intervention. Os idiomas considerados para a busca foram o português, o inglês e o espanhol.

Foram incluídos no estudo: artigos originais, ensaios clínicos randomizados, não randomizados, estudos do tipo coorte e estudos disponibilizados na íntegra. E excluídos: teses, dissertações, estudos que não citassem nenhuma intervenção farmacêutica, e pesquisas realizadas por acadêmicos de farmácia ou com técnicos de farmácia. 


\section{Resultados}

Foram encontrados um total de 1.125 trabalhos, a partir da pesquisa nos bancos de dados. A Figura 1 demonstra, através do fluxograma, o método de seleção utilizado para escolha dos estudos. Destes, 16 foram considerados para o estudo. E estão listados no Quadro 1, em que é possível observar elementos importantes dos estudos, como o ano de publicação, os autores, o título, o tipo de estudo, o objetivo, os resultados obtidos e a conclusão.

Figura 1: fluxograma do método de seleção dos trabalhos.

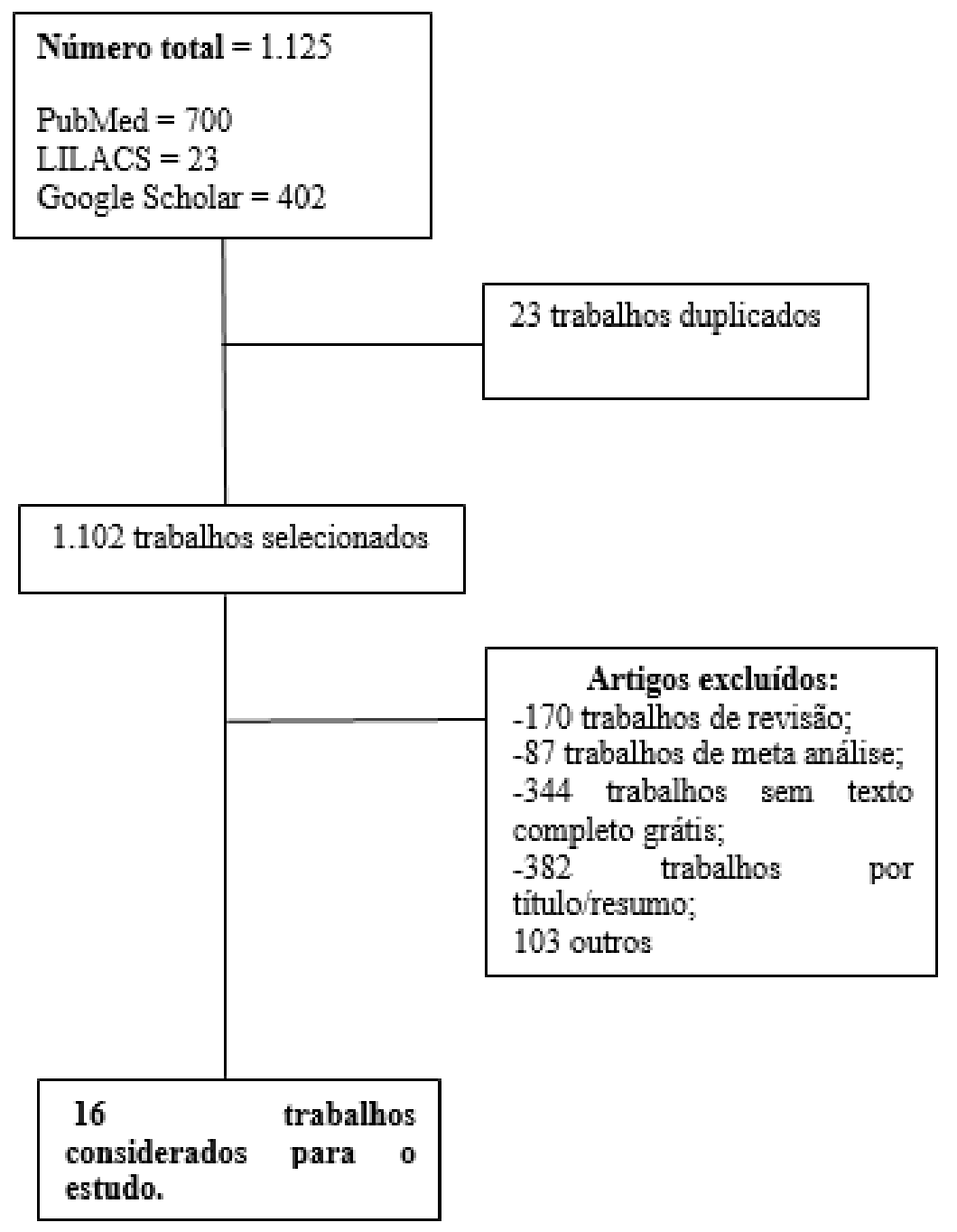

Fonte: Autores. 
Research, Society and Development, v. 10, n. 16, e316101623753, 2021

(CC BY 4.0) | ISSN 2525-3409 | DOI: http://dx.doi.org/10.33448/rsd-v10i16.23753

\begin{tabular}{|c|c|c|c|c|c|c|}
\hline \multicolumn{7}{|c|}{ Quadro 1: Trabalhos Incluídos na Revisão. } \\
\hline ANO & AUTORES & TÍTULO & $\begin{array}{l}\text { TIPO DE } \\
\text { ESTUDO }\end{array}$ & OBJETIVOS & RESULTADOS & CONCLUSÃO \\
\hline 2012 & $\begin{array}{l}\text { Plaster et } \\
\text { al. }\end{array}$ & $\begin{array}{l}\text { Reduction of cardiovascular risk in } \\
\text { patients with metabolic syndrome in a } \\
\text { community health center after a } \\
\text { pharmaceutical care program of } \\
\text { pharmacotherapy follow-up }\end{array}$ & $\begin{array}{l}\text { Ensaio clínico } \\
\text { controlado e } \\
\text { randomizado }\end{array}$ & $\begin{array}{l}\text { O objetivo foi determinar o } \\
\text { impacto de um programa de } \\
\text { cuidado farmacêutico em } \\
\text { pacientes com síndrome } \\
\text { metabólica. }\end{array}$ & $\begin{array}{l}\text { Entre os pacientes com SM, } 100 \% \text { faziam uso de medicamentos } \\
\text { para diminuir aglicose, anti-hipertensivos (GC: } 72 \% \text {; GI: } 73 \% \text { ) e } \\
\text { hipoglicemiantes (GC: } 12.0 \% \text {; GI: } 14.7 \%) \text {. Apenas } 20,7 \% \text { do GI } \\
\text { foram considerados aderentes aos fármacos prescritos. No GC } \\
\text { foi observado aumento do risco de Doença Arterial Coronariana } \\
\text { (DAC) (22 } 2 \text { para } 26 \pm 3 ; \mathrm{p}<0,05) \text {, enquanto no GI foi observado } \\
\text { redução (22 } 22 \text { para } 14 \pm 2 \% ; \mathrm{p}<0,01)\end{array}$ & $\begin{array}{l}\text { O programa melhora o funcionamento do } \\
\text { serviço e acarreta uma melhora clínica, } \\
\text { com redução de risco cardiovascular e } \\
\text { com uma melhora na adesão. }\end{array}$ \\
\hline 2013 & $\begin{array}{l}\text { Saleem et } \\
\text { al. }\end{array}$ & $\begin{array}{l}\text { Pharmacist intervention in improving } \\
\text { hypertension related knowledge, } \\
\text { treatment medication adherence and } \\
\text { health-related quality of life: a non- } \\
\text { clinical randomized controlled trial }\end{array}$ & $\begin{array}{l}\text { Estudo } \\
\text { randomizado }\end{array}$ & $\begin{array}{l}\text { Avaliar se o cuidado } \\
\text { farmacêutico pode melhorar a } \\
\text { compreensão sobre a } \\
\text { hipertensão, melhorar adesão } \\
\text { ao tratamento e a qualidade de } \\
\text { vida em geral. }\end{array}$ & $\begin{array}{l}\text { Houve um aumento significativo nos níveis de conhecimento dos } \\
\text { participantes sobre hipertensão e adesão medicamentosa entre o } \\
\text { grupo intervencionista após completar a intervenção. Pressão } \\
\text { sistólica e diastólica significativamente mais baixa, com níveis } \\
\text { de pressão mais baixos também foram observados entre o grupo } \\
\text { intervencionista. }\end{array}$ & $\begin{array}{l}\text { Houve aumento significativo } \\
\text { compreensão acerca da doença, no } \\
\text { controle da pressão e na adesão ao } \\
\text { tratamento. }\end{array}$ \\
\hline 2013 & $\begin{array}{l}\text { Tommelein } \\
\text { et al. }\end{array}$ & $\begin{array}{l}\text { Effectiveness of pharmaceutical care } \\
\text { for patients with chronic obstructive } \\
\text { pulmonar disease (PHARMACOP): a } \\
\text { randomized controlled trial }\end{array}$ & $\begin{array}{l}\text { Estudo } \\
\text { randomizado }\end{array}$ & $\begin{array}{lll}\text { Avaliar a } & \text { eficácia } & \text { de um } \\
\text { programa } & \text { de } & \text { cuidado } \\
\text { farmacêutico } & \text { para } & \text { pacientes } \\
\text { com DPOC. } & & \end{array}$ & $\begin{array}{l}\text { No final do ensaio, a pontuação de inalação [diferença média } \\
\text { estimada (A), 13,5\%; Intervalo de confiança de } 95 \% \text { (IC), } 10,8- \\
16,1 ; \mathrm{P}<0,0001] \text { e adesão à medicação }(\Delta, 8,51 \% \text {; IC de } 95 \% \text {, } \\
4,63-12,4 ; \mathrm{P}<0,0001) \text { foram significativamente maiores no } \\
\text { grupo de intervenção em comparação com o grupo de controle. }\end{array}$ & $\begin{array}{l}\text { Houve melhora no regime } \\
\text { farmacoterapêutico dos pacientes, com } \\
\text { um aumento na adesão ao tratamento. }\end{array}$ \\
\hline 2014 & $\begin{array}{l}\text { Chung et } \\
\text { al. }\end{array}$ & $\begin{array}{l}\text { Effects of a pharmaceutical care } \\
\text { model on medication adherence and } \\
\text { glycemic control of people with type } \\
2 \text { diabetes }\end{array}$ & $\begin{array}{l}\text { Estudo } \\
\text { randomizado }\end{array}$ & $\begin{array}{l}\text { Avaliar os efeitos de um } \\
\text { modelo } \\
\text { farmacêutico na adesão à } \\
\text { medicação e nos níveis } \\
\text { glicêmicos de pessoas com } \\
\text { diabetes mellitus 2. }\end{array}$ & $\begin{array}{l}\text { Diferenças estatisticamente significativas nos valores de FBG e } \\
\text { hemoglobina glicada foram observadas entre os grupos de } \\
\text { controle e intervenção após o fornecimento de PC (mediana de } \\
\text { FBG, 9,0 versus } 7,2 \text { mmol / L [P } \square 0,001] \text {; nível mediano de } \\
\text { hemoglobina glicada, 9,1\% versus } 8,0 \% \text { [P } \square 0,001] \text { em } 12 \\
\text { meses). A adesão à medicação também foi significativamente } \\
\text { associada ao fornecimento de CP, com maior proporção no } \\
\text { grupo de intervenção do que no grupo controle ( } 75,0 \% \text { versus } \\
58,7 \% ; \mathrm{P}=0,007) \text {. }\end{array}$ & $\begin{array}{l}\text { O cuidado farmacêutico resultou em } \\
\text { melhora da adesão, bem como no } \\
\text { controle glicêmico. }\end{array}$ \\
\hline 2015 & Modé et al. & $\begin{array}{l}\text { Atenção farmacêutica em pacientes } \\
\text { hipertensos: um estudo piloto }\end{array}$ & $\begin{array}{l}\text { Estudo } \\
\text { randomizado }\end{array}$ & $\begin{array}{l}\text { Implantar um estudo piloto de } \\
\text { cuidado farmacêutico em uma } \\
\text { farmácia privada, para } \\
\text { pacientes hipertensos. }\end{array}$ & $\begin{array}{l}\text { Dentre os } 20 \text { pacientes que participaram do estudo, } 45 \% \\
\text { apresentaram PA descontrolada }(\geq 140 \times 90 \mathrm{mmHg}) \text { na primeira } \\
\text { entrevista. Após as intervenções farmacêuticas o número foi } \\
\text { reduzido para } 20 \% \text {. No grupo intervenção, a média da PA } \\
\text { sistólica e a da PA diastólica apresentou redução } \\
\text { respectivamente de } 17 \mathrm{mmHg} \text { e 8mmHg. }\end{array}$ & $\begin{array}{l}\text { O cuidado farmacêutico promoveu uma } \\
\text { otimização dos resultados terapêuticos } \\
\text { com aumento na adesão, após maiores } \\
\text { esclarecimentos sobre a doença. }\end{array}$ \\
\hline 2015 & $\begin{array}{l}\text { Rigoni et } \\
\text { al. }\end{array}$ & $\begin{array}{l}\text { Pharmacotherapy review: a proposal } \\
\text { to improve a medication adherence } \\
\text { among hypertensive patients }\end{array}$ & $\begin{array}{l}\text { Estudo } \quad \text { de } \\
\text { intervenção não } \\
\text { controlado }\end{array}$ & $\begin{array}{l}\text { Avaliar a influência da revisão } \\
\text { da terapia na adesão aos } \\
\text { medicamentos e no controle da } \\
\text { hipertensão. }\end{array}$ & $\begin{array}{l}\text { Houve aumento significativo da taxa de adesão, tanto pelo teste } \\
\text { de Morisky-Green } \quad(p<0,001) \text { quanto pela avaliação } \\
\text { autorreferida }(p=0,004) \text {. Também houve melhora nos níveis de } \\
\text { pressão arterial sistólica }(p<0,001) \text { e diastólica }(p=0,002) \text { e no } \\
\text { número de pacientes com hipertensão controlada }(p=0,006) \text {. }\end{array}$ & 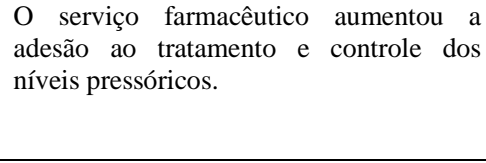 \\
\hline 2015 & Xin et al. & $\begin{array}{l}\text { Effect of pharmaceutical care on } \\
\text { medication adherence of patients } \\
\text { newly prescribed insulin therapy: a } \\
\text { randomized controlled study }\end{array}$ & $\begin{array}{l}\text { Estudo } \\
\text { randomizado }\end{array}$ & $\begin{array}{l}\text { Avaliar a eficácia do cuidado } \\
\text { farmacêutico na adesão de } \\
\text { pacientes com terapia insulínica } \\
\text { recém prescrita. }\end{array}$ & $\begin{array}{l}\text { Após as intervenções de } 12 \text { meses, melhorias significativas na } \\
\text { adesão à medicação foram verificadas para o grupo de } \\
\text { assistência farmacêutica de acordo com o teste de Morisky- } \\
\text { Green ( } 50,8 \% \text { dos pacientes aderentes no início do estudo vs } \\
80,7 \% \text { dos pacientes aderentes após as intervenções de } 12 \text { meses; }\end{array}$ & $\begin{array}{l}\text { O cuidado farmacêutico teve um } \\
\text { resultado benéfico sobre o aumento na } \\
\text { adesão da terapia insulínica recém } \\
\text { prescrita. }\end{array}$ \\
\hline
\end{tabular}


Research, Society and Development, v. 10, n. 16, e316101623753, 2021

(CC BY 4.0) | ISSN 2525-3409 | DOI: http://dx.doi.org/10.33448/rsd-v10i16.23753

\begin{tabular}{|c|c|c|c|c|c|c|}
\hline & & & & & $\begin{array}{l}\text { P <0,01 ), enquanto nenhuma mudança significativa foi } \\
\text { verificada no grupo de controle }\end{array}$ & \\
\hline 2017 & $\begin{array}{l}\text { Azevedo et } \\
\text { al. }\end{array}$ & $\begin{array}{l}\text { Effectiveness of home } \\
\text { pharmaceutical interventions in } \\
\text { metabolic syndrome: a randomized } \\
\text { controlled trial }\end{array}$ & $\begin{array}{l}\text { Estudo } \\
\text { randomizado }\end{array}$ & $\begin{array}{l}\text { Avaliar a eficácia das } \\
\text { intervenções farmacêuticas, em } \\
\text { domicílio, para pacientes com } \\
\text { síndrome metabólica. }\end{array}$ & $\begin{array}{l}\text { A amostra foi composta por } 63 \text { indivíduos ( } 33 \text { GI e } 30 \text { GC) e } \\
\text { maiores de } 60 \text { anos. A maioria das intervenções farmacêuticas } \\
\text { foi educacional e / ou comportamental. No grupo de intervenção, } \\
\text { diferenças significativas foram observadas nos parâmetros de } \\
\text { pressão sistólica e diastólica triglicérides, adesão à medicação e } \\
\text { PRM. }\end{array}$ & $\begin{array}{l}\text { As intervenções farmacêuticas, em casa, } \\
\text { melhoraram a adesão, reduziram os } \\
\text { Problemas Relacionados ao Medicamento } \\
\text { e ajudou a controlar os componentes da } \\
\text { síndrome. }\end{array}$ \\
\hline 2017 & $\begin{array}{l}\text { Gerenutti et } \\
\text { al. }\end{array}$ & $\begin{array}{l}\text { The Effectiveness of a } \\
\text { Pharmaceutical Care Model on } \\
\text { Adherence to Antiretroviral Therapy: } \\
\text { A SAME-Based Cohort Study in } \\
\text { Brazil }\end{array}$ & $\begin{array}{l}\text { Estudo original } \\
\text { de coorte }\end{array}$ & $\begin{array}{l}\text { Verificar a eficácia de um } \\
\text { modelo } \text { de cuidado } \\
\text { farmacêutico sobre adesão a } \\
\text { terapia antirretroviral em } \\
\text { pacientes com HIV }\end{array}$ & $\begin{array}{l}\text { Maiores taxas de adesão foram observadas no grupo de } \\
\text { intervenção }(p<0,05) \text {. O uso de outras drogas não influenciou na } \\
\text { adesão à TARV }(p=0,30) \text {. Houve correlação positiva entre a } \\
\text { adesão e o percentual de pacientes do grupo intervenção com } \\
\text { cargas virais indetectáveis }(p=0,0004) \text { e níveis mais elevados } \\
\text { de linfócitos CD4 }(p=0,0024) \text {. }\end{array}$ & $\begin{array}{l}\text { O modelo promoveu uma melhora na } \\
\text { adesão ao tratamento, bem como } \\
\text { resultados clínicos. }\end{array}$ \\
\hline 2017 & $\begin{array}{l}\text { Haramiova } \\
\text { et al. }\end{array}$ & $\begin{array}{l}\text { The effectiveness of daily SMS } \\
\text { reminders in pharmaceutical care of } \\
\text { older adults onimproving patients' } \\
\text { adherencer toantihypertensive } \\
\text { medication (SPPA): studyprotocol for } \\
\text { a randomized controlled trial }\end{array}$ & $\begin{array}{l}\text { Estudo } \\
\text { randomizado }\end{array}$ & $\begin{array}{l}\text { Avaliar se lembretes diários por } \\
\text { SMS, enviados por por } \\
\text { farmacêuticos, além da } \\
\text { assistência farmacêutica padrão } \\
\text { melhora a adesão ao tratamento } \\
\text { anti hipertensivo em idosos } \\
\text { ambulatoriais }\end{array}$ & $\begin{array}{l}\text { Os resultados incluem: mudança no MMAS-8; comparação das } \\
\text { taxas de adesão usando contagem de comprimidos; mudança na } \\
\text { pressão sistólica e satisfação do paciente. }\end{array}$ & $\begin{array}{l}\text { Houve melhora na adesão, efeitos sobre } \\
\text { os custos do tratamento e relato de } \\
\text { satisfação dos pacientes. }\end{array}$ \\
\hline 2017 & $\begin{array}{ll}\text { Marfo } & \mathrm{e} \\
\text { Daaku }\end{array}$ & $\begin{array}{l}\text { Exploring the extended role of the } \\
\text { community pharmacist in improving } \\
\text { blood pressure control among } \\
\text { hypertensivepatients in a developing } \\
\text { setting }\end{array}$ & $\begin{array}{l}\text { Estudo não } \\
\text { randomizado }\end{array}$ & $\begin{array}{l}\text { Avaliar o efeito de um modelo } \\
\text { de cuidado farmacêutico na } \\
\text { adesão ao tratamento e controle } \\
\text { da pressão arterial. }\end{array}$ & $\begin{array}{l}\text { A diferença média da pressão arterial diastólica entre o grupo } \\
\text { intervenção e o grupo controle foi estatisticamente significativa } \\
(p=0,001) \text {. A diferença média de adesão entre os dois grupos } \\
\text { também foi estatisticamente significativa no final do estudo. }(\mathrm{p}= \\
0,001) \text {. }\end{array}$ & $\begin{array}{l}\text { A intervenção farmacêutica resultou na } \\
\text { melhora da adesão e na melhora da } \\
\text { pressão diastólica. }\end{array}$ \\
\hline 2018 & Lu et al. & $\begin{array}{l}\text { Impact of multidisciplinary } \\
\text { collaborative pharmaceutical care on } \\
\text { knowledge, adherence, and efficacy } \\
\text { of hormone therapy in climacteric } \\
\text { women }\end{array}$ & $\begin{array}{l}\text { Estudo } \\
\text { randomizado }\end{array}$ & $\begin{array}{l}\text { Avaliar o impacto do cuidado } \\
\text { farmacêutico no conhecimento, } \\
\text { adesão e eficácia da terapia } \\
\text { hormonal em mulheres no } \\
\text { climatério }\end{array}$ & $\begin{array}{l}\text { O grupo de intervenção, que recebeu CP, apresentou } \\
\text { conhecimento, adesão e eficácia significativamente maiores e } \\
\text { melhores do que o grupo controle, demonstrando a eficácia do } \\
\text { CP fornecido. Os escores de conhecimento dos grupos de } \\
\text { intervenção e controle nos meses } 3,6 \text { e } 12 \text { foram ( }(73,12 \text { vs } \\
59,28),(77,63 \text { vs } 66,19) \text { e }(80,81 \text { vs } 66,64) \text {; os dados para adesão } \\
\text { foram (90,97\% vs } 82,17 \%) \text {, ( } 93,21 \% \text { vs } 87,79 \%) \text { e }(95,81 \% \text { vs } \\
93,38 \%) \text {; os valores do Índice de Kupperman modificado } \\
\text { foram (17,15 vs } 24,05),(13,22 \text { vs } 22,01) \text { e }(12,21 \text { vs } 23,15) \text {, } \\
\text { respectivamente. }\end{array}$ & $\begin{array}{l}\text { O cuidado farmacêutico melhorou o } \\
\text { conhecimento, a adesão e a eficácia do } \\
\text { tratamento hormonal. }\end{array}$ \\
\hline 2018 & Wuyts et al. & $\begin{array}{l}\text { Studying the impact of a medication } \\
\text { use evaluation for polymedicated } \\
\text { older patients by the community } \\
\text { pharmacist (SIMENON): study } \\
\text { protocol }\end{array}$ & $\begin{array}{l}\text { Estudo } \\
\text { randomizado }\end{array}$ & $\begin{array}{l}\text { Investigar o efeito da revisão } \\
\text { dos medicamentos, por } \\
\text { farmacêuticos, para pacientes } \\
\text { idosos } \quad \text { ambulatoriais } \\
\text { polimedicados }\end{array}$ & $\begin{array}{l}\text { Os resultados incluem adesão, autocuidado, aumento na } \\
\text { satisfação do paciente, e diminuiçãa dos incidentes de queda e } \\
\text { uso de serviços de saúde de emergência. }\end{array}$ & $\begin{array}{l}\text { A revisão promoveu uma diminuição nos } \\
\text { problemas relacionados a medicamentos, } \\
\text { com melhora na adesão ao tratamento. }\end{array}$ \\
\hline 2019 & $\begin{array}{l}\text { Chambela } \\
\text { et al. }\end{array}$ & $\begin{array}{l}\text { Impact of pharmaceutical care on the } \\
\text { quality of life of patients with heart } \\
\text { failure due to chronic Chagas disease: } \\
\text { Randomized clinical trial }\end{array}$ & $\begin{array}{l}\text { Estudo } \\
\text { randomizado }\end{array}$ & $\begin{array}{l}\text { Avaliar se o ruidado } \\
\text { farmacêutico melhora a } \\
\text { qualidade de vida de indivíduos } \\
\text { com doença de chagas e } \\
\text { insuficiência cardíaca. }\end{array}$ & $\begin{array}{l}\text { A adesão ao tratamento médico aumentou logo após } 3 \text { meses de } \\
\text { acompanhamento e a incidência de PRM diminuiu após } 6 \text { meses } \\
\text { de acompanhamento apenas no grupo PC. }\end{array}$ & $\begin{array}{l}\text { Houve diminuição dos problemas } \\
\text { relacionados aos medicamentos, aumento } \\
\text { da qualidade de vida e melhora na } \\
\text { adesão. }\end{array}$ \\
\hline
\end{tabular}


Research, Society and Development, v. 10, n. 16, e316101623753, 2021

(CC BY 4.0) | ISSN 2525-3409 | DOI: http://dx.doi.org/10.33448/rsd-v10i16.23753

\begin{tabular}{|c|c|c|c|c|c|c|}
\hline 2019 & $\begin{array}{l}\text { Khdour et } \\
\text { al. }\end{array}$ & $\begin{array}{l}\text { Pharmaceutical care for adult asthma } \\
\text { patients: A controlled intervention } \\
\text { one-year follow-up study }\end{array}$ & $\begin{array}{l}\text { Estudo } \\
\text { randomizado }\end{array}$ & $\begin{array}{lccc}\text { Implementar } & \text { e } & \text { avaliar } & \text { um } \\
\text { serviço } & \text { de } & \text { cuidado } \\
\text { farmacêutico } & \text { hospitalar para } \\
\text { pacientes asmáticos } & \end{array}$ & $\begin{array}{l}\text { Ao longo do período de acompanhamento de } 12 \text { meses, uma } \\
\text { diferença significativa foi observada entre os grupos de } \\
\text { intervenção e controle com relação ao controle da asma ( } 38,2 \% \\
\text { vs } 10,0 \% \text {; P <0,001), técnica de inalação correta média } \\
\text { (intervalo de confiança [IC ]: } 8,1,7,8-8,5 \text { vs IC: } 6,1 ; 5,6-6,6 ; \mathrm{P}= \\
0,01) \text { e boa adesão à medicação }(60,7 \% \text { vs } 50,0 \%, \mathrm{P}=0,02) \text {. }\end{array}$ & $\begin{array}{l}\text { O cuidado farmacêutico favoreceu um } \\
\text { aumento na adesão, bem como no } \\
\text { controle da asma. }\end{array}$ \\
\hline 2019 & Naqvi et al. & $\begin{array}{l}\text { Impact of pharmacist educational } \\
\text { intervention on disease knowledge, } \\
\text { rehabilitation and medication } \\
\text { adherence, treatment-induced direct } \\
\text { cost, health related quality of life and } \\
\text { satisfaction in patients with } \\
\text { rheumatoid arthritis: study protocol } \\
\text { for a randomized controlled trial }\end{array}$ & $\begin{array}{l}\text { Estudo } \\
\text { randomizado }\end{array}$ & $\begin{array}{l}\text { Avaliar a eficácia do cuidado } \\
\text { farmacêutico no conhecimento } \\
\text { sobre a doença, adesão ao } \\
\text { tratamento, melhora na } \\
\text { qualidade de vida e efeitos } \\
\text { sobre os custos no tratamento. }\end{array}$ & $\begin{array}{l}\text { Os resultados incluem mudança na pontuação média desde o } \\
\text { início (semana 0) e no acompanhamento (semana 12) em } \\
\text { conhecimento da doença, adesão a medicamentos e } \\
\text { reabilitação/fisioterapia. Mudanças no custo direto médio do } \\
\text { tratamento, QVRS e satisfação do paciente com o } \\
\text { aconselhamento farmacêutico. }\end{array}$ & $\begin{array}{l}\text { O cuidado farmacêutico promoveu um } \\
\text { aumento no conhecimento dos pacientes } \\
\text { a respeito da doença, melhorou a adesão e } \\
\text { a qualidade de vida, e nos custos do } \\
\text { tratamento. E foi reconhecido } \\
\text { positivamente pelos pacientes. }\end{array}$ \\
\hline
\end{tabular}

Fonte: Autores. 
Research, Society and Development, v. 10, n. 16, e316101623753, 2021

(CC BY 4.0) | ISSN 2525-3409 | DOI: http://dx.doi.org/10.33448/rsd-v10i16.23753

Entre os trabalhos inclusos, observou-se que o tipo de estudo preponderante é o randomizado, e que a distribuição ocorre da seguinte forma: 13 são do tipo randomizado, 2 não controlados e 1 estudo do tipo coorte. Plaster et al. (2012), Saleem et al. (2013), Tommelein et al. (2013), Chung et al. (2014), Modé et al. (2015), Xin et al. (2015), Azevedo et al. (2017), Haramiova et al. (2017), Lu et al. (2018), Wuyts et al. (2018), Khdour et al. (2019), Naqvi et al. (2019) e Chambela et al. (2019) representam o tipo de estudo randomizado. Enquanto Rigoni et al. (2015), e Marfo e Daaku (2017), o tipo não controlado. E Gerenutti et al. (2017), o estudo do tipo coorte.

Já com relação aos cenários em que os trabalhos foram desenvolvidos, observou-se uma certa pluralidade, e que incluiu: farmácias comunitárias, hospitais, unidades básicas de saúde pública e serviços de atenção especializada. Destes, 6 foram desenvolvidos em farmácias comunitárias: Tommelein et al. (2013), Modé et al. (2015), Rigoni et al. (2015), Haramiova et al. (2017), Marfo e Daaku (2017), e Wuyts et al. (2018); 5 em hospitais: Saleem et al. (2013), Chung et al. (2014), Xin et al. (2015), Lu et al. (2018), e Khdour et al. (2019); 2 em unidades básicas de saúde pública: Plaster et al. (2012) e Azevedo et al. (2017); e 3 em serviços de atenção especializada: Gerenutti et al. (2017), Chambela et al. (2019) e Naqvi et al. (2019).

\subsection{O cuidado farmacêutico aplicado à diferentes condições clínicas para melhoria da adesão}

A partir da análise e sumarização dos trabalhos levantados, identificou-se uma pluralidade de condições clínicas, para as quais o cuidado farmacêutico foi empregado, como opção, para melhorar e aumentar a adesão ao tratamento medicamentoso.

\subsubsection{Hipertensão}

Haramiova et al. (2017), Marfo e Daaku (2017), Modé et al. (2015), Rigoni et al. (2015), e Saleem et al. (2013), retratam em seus trabalhos a utilização do cuidado farmacêutico como ferramenta para aumentar a adesão ao tratamento anti hipertensivo e minimizar os riscos primários e secundários advindos de tal doença.

\subsubsection{Diabetes}

Chung et al. (2014) e Xin et al. (2015), descrevem em seus estudos o envolvimento de pacientes diabéticos com o farmacêutico, a partir do cuidado farmacêutico, para melhorar a adesão tanto ao tratamento com medicamentos orais, como com insulinoterapia.

\subsubsection{Síndrome metabólica}

Plaster et al. (2012), e Azevedo et al. (2017) abordam em estudo a participação do farmacêutico na vida de pacientes com síndrome metabólica e associam o cuidado farmacêutico com um aumento na adesão ao tratamento.

\subsubsection{Asma}

Dentre os trabalhos analisados, Khdour et al. (2019), descreve e avalia como objeto de estudo, o cuidado farmacêutico como intervenção para potencializar a adesão em indivíduos asmáticos.

\subsubsection{Doença Pulmonar Obstrutiva Crônica (DPOC)}

A eficácia da interação entre o farmacêutico e pacientes com DPOC, a partir do cuidado farmacêutico, no processo de adesão, é abordada por Tommelein et al. (2013), em estudo. 


\subsubsection{HIV/AIDS}

Gerenutti et al. (2017), cita em trabalho a participação do farmacêutico na melhora da adesão da terapia antirretroviral, a partir da realização do cuidado farmacêutico.

\subsubsection{Artrite reumatoide}

Naqvi et al. (2019), retratam a atuação do profissional farmacêutico a partir do manejo de pacientes com artrite reumatoide através do cuidado farmacêutico, como forma de aumentar a adesão ao tratamento, o conhecimento sobre a doença e a qualidade de vida dos pacientes.

\subsubsection{Doença de chagas}

A prestação do cuidado farmacêutico a pacientes chagásicos para promoção da qualidade de vida e melhora na adesão ao tratamento é citado por Chambela et al. (2019).

\subsubsection{Climatério}

Lu et al. (2018), trazem em estudo a abordagem do cuidado farmacêutico direcionado para mulheres em climatério, e o desenvolvimento de conhecimentos e adesão à terapia hormonal.

\subsubsection{Polimedicação}

Wuyts et al. (2018), relatam a participação do profissional farmacêutico na melhora da adesão e diminuição do uso de serviços de emergência por idosos, a partir da prestação do cuidado farmacêutico.

\subsection{Intervenções farmacêuticas}

Diferentes formas de intervenções foram realizadas, a partir do cuidado farmacêutico, e resultaram na promoção de uma melhora na adesão ao tratamento. A combinação de mais de um tipo de intervenção também foi observado entre os diferentes trabalhos analisados.

\subsubsection{Educação em Saúde}

Azevedo et al. (2017), Chung et al. (2014), Khdour et al. (2019), Lu et al. (2018), Marfo e Daaku (2017), Naqvi et al. (2019), Plaster et al. (2012), Saleem et al. (2013), Tommelein et al. (2013), e Xin et al. (2015), apontaram, em estudo, a atuação do farmacêutico a partir da educação em saúde, para melhoria da adesão ao tratamento.

A partir de tais estudos, identificou-se que a prática da educação em saúde ocorreu tanto de forma verbal como a partir da entrega de materiais didáticos e informativos, como cartilhas e adesivos personalizados para colocar nos frascos dos medicamentos.

Os pacientes foram educados quanto a informações sobre a doença de origem, a forma como tomar os medicamentos, possíveis interações entre medicamento-medicamento e medicamento-alimento, os efeitos adversos que poderiam surgir decorrentes do tratamento, a forma de aplicação de dispositivos, como a insulina, e de utilização, como o medidor de glicose e a técnica de inalação. Além da educação voltada para prevenção, a partir da abordagem de aspectos relacionados aos hábitos e estilos de vida. 


\subsubsection{Acompanhamento por aparelho telefônico}

Chung et al. (2014), Khdour et al. (2019), Modé et al. (2015), e Xin et al. (2015), descreveram como estratégia de intervenção para melhorar a adesão, o acompanhamento dos pacientes por telefone, pelo profissional farmacêutico.

Os trabalhos evidenciaram que a realização do telefonema era acompanhada de perguntas estratégicas e repasse de informações importantes sobre o tratamento, e que tem como objetivo ajudar a esclarecer qualquer dúvida ou problema que surja em decorrência dos medicamentos utilizados.

O acompanhamento consistia na verificação sobre a condição atual do paciente, e se o mesmo se encontrava bem, informações sobre efeitos adversos e como manejá-los, caso aparecessem, e repasse de informações sobre o tratamento e como realizá-lo.

\subsubsection{Acompanhamento farmacoterapêutico}

Chambela et al. (2019), e Plaster et al. (2012), registraram o acompanhamento farmacoterapêutico como intervenção do farmacêutico, capaz de potencializar a adesão medicamentosa.

O acompanhamento farmacoterapêutico ocorreu a partir da utilização do método Dáder, composto por nove etapas, que vai desde a oferta do serviço até a realização de visitas sucessivas. Durante o acompanhamento o farmacêutico realizou a explicação do esquema terapêutico, de cada medicamento e da prescrição, e relatou a importância de seguir o tratamento e da adesão medicamentosa. Além de identificar e resolver os Problemas Relacionados a Medicamentos (PRM).

\subsubsection{Revisão dos medicamentos}

Azevedo et al. (2017), Chung et al. (2014), Modé et al. (2015), Rigoni et al. (2015), e Wuyts et al. (2018), utilizaram como forma de intervenção e cuidado farmacêutico, a prática da revisão dos medicamentos para suporte na adesão ao tratamento medicamentoso.

A revisão de medicamentos foi realizada de forma que possibilitou a identificação dos PRM e a possível correção dos mesmos, a partir de diferentes estratégias, como medidas educativas, a adição de medicamentos, a substituição de medicamentos e a alteração na frequência de dose.

\subsubsection{Lembretes por Serviço de Mensagem de Texto}

Haramiova et al. (2017), destacam a utilização de lembretes por mensagens de texto, enviadas por farmacêuticos, como intervenção para melhorar a aderência ao tratamento medicamentoso.

As mensagens de texto tiveram como objetivo lembrar os pacientes de tomar os seus medicamentos. Além de fornecer informações sobre o seu tratamento. Os lembretes foram formulados, de forma personalizada, de acordo com as informações que estão contidas nas prescrições dos pacientes, como o nome dos medicamentos, dosagens, frequência de utilização, e outras informações que fossem de interesse.

\section{Discussão}

\subsection{O cuidado farmacêutico aplicado à diferentes condições clínicas para melhoria da adesão}

A partir dos resultados, observa-se que a condição clínica a qual o cuidado farmacêutico foi mais aplicado foi a hipertensão, seguida do diabetes. As demais patologias assemelharam-se com relação ao número de trabalhos publicados, em que o cuidado farmacêutico foi citado como abordagem para melhorar a aderência ao tratamento.

De acordo com Araujo et al. (2017), o conceito de adesão e a sua ocorrência, está diretamente associado com a relação 
estabelecida entre o paciente e o profissional de saúde, na qual o paciente deve ser um colaborador ativo e assumir um papel central no processo de cuidado.

Ainda para Araujo et al. (2017), a adesão é algo complexo, já que o processo de tratamento não é limitado a terapêutica farmacológica e ao seguimento do que está prescrito pelos profissionais de saúde.

Segundo Oliveira et al. (2015), a adesão decorre de um agrupamento de situações que envolvem as crenças dos pacientes, o suporte familiar, a relação entre o profissional e o paciente, aspectos relacionados a doença, ao tratamento e o acesso aos medicamentos e serviços de saúde. De acordo com a OMS (2003), cinco dimensões influenciam na adesão ao tratamento, a partir da multifatorialidade do processo: fatores relacionados a questões socioeconômicas, ao paciente, a doença, ao tratamento e aos serviços de saúde.

Gewehr et al. (2018), define que a não adesão ao tratamento medicamentoso ocorre quando há desistência do uso dos medicamentos, de forma autônoma, sem acompanhamento médico, ou, quando o tratamento é realizado de forma irregular, a partir de alterações nos intervalos de tomada das doses, e através de uma utilização terapêutica não uniforme dos medicamentos.

Segundo Menditto et al. (2020), a falta de adesão ao tratamento medicamentoso interfere negativamente na evolução das condições clínicas e na eficácia do tratamento. Já a OMS (2003), destaca que a má adesão compromete a terapia, o panorama clínico e a qualidade de vida.

Holt et al. (2010), relatam que um dos principais fatores que contribui para o controle inadequado da pressão arterial em pacientes com hipertensão, é a baixa adesão ao tratamento medicamentoso. Segundo Ho et al. (2009), a baixa adesão ao tratamento anti hipertensivo é considerada como um dos grandes interferentes para o controle e manutenção da pressão arterial em níveis satisfatórios.

Conforme Xin et al. (2015), a baixa adesão a insulinoterapia promove uma elevação no número de internações hospitalares e uma redução na qualidade de vida de pacientes portadores de diabetes mellitus. Já Chung et al. (2014), definem que os principais determinantes para o sucesso da terapia no controle do diabetes são a mudança nos hábitos de vida e a adesão aos medicamentos antidiabéticos.

Plaster et al. (2012), estipulam que uma condição essencial para o controle de DCNT, como a síndrome metabólica, é a presença da adesão ao tratamento farmacológico. Khdour et al. (2019), relatam que as dificuldades acerca da gestão da asma podem estar, parcialmente, atreladas a não adesão aos medicamentos e ao uso impróprio de inaladores.

Tommelein et al. (2013), destacam como parte fundamental da gestão da DPOC, recomendada pela Iniciativa Global para Doença Pulmonar Obstrutiva Crônica, a garantia da adesão ao tratamento e o conhecimento, por parte dos pacientes, sobre a técnica de inalação e o uso de inaladores. Para Martin et al. (2017), o êxito no tratamento do HIV é dependente da adesão a terapia antirretroviral, já que a baixa adesão promove piora no quadro clínico, redução da eficácia dos medicamentos e disseminação de cepas de HIV resistentes.

Hernández et al. (2020), citam em estudo que a presença da polifarmácia, e o uso simultâneo de cinco ou mais medicamentos, é uma condição que interfere, negativamente, em diferentes níveis da adesão ao tratamento, e que é considerada como um fator de risco para a segurança do paciente.

Conforme a OMS (2003), quando há adesão, há um impacto positivo sobre a condição de saúde e desfecho clínico do paciente, menor uso dos serviços de saúde em função de piora nos quadros clínicos, e consequentemente, vantagens para o setor econômico, além de melhora na qualidade de vida dos pacientes. Oliveira et al. (2015), apontam que quando a má adesão ao tratamento medicamentoso ocorre, o número de complicações pode crescer, e resultar no aumento do número de hospitalizações e na elevação dos custos ao sistema de saúde. 
Diferentes estratégias e profissionais de saúde estão envolvidos no processo de promover a adesão ao tratamento. Xin et al. (2015), destaca que os farmacêuticos clínicos são profissionais que estão bem posicionados quanto a atuação direcionada para superar as barreiras de adesão, e melhorar a aderência dos pacientes ao tratamento medicamentoso. Heisler et al. (2012), relatam que vários estudos demonstraram a importância do cuidado farmacêutico na melhora da adesão ao tratamento, no autocuidado e, em alguns casos, em retorno econômico líquido.

\subsection{Intervenções farmacêuticas}

O panorama da profissão farmacêutica sofre uma reformulação quando surge na década de 1960 a farmácia clínica, que promoveu a reaproximação do profissional farmacêutico com o paciente, e a atenção farmacêutica, ou também como é conhecido, o cuidado farmacêutico, cujo objetivo foi direcionado a promoção, proteção e recuperação da saúde, tanto individual quanto coletiva, a partir do uso racional dos medicamentos (Agostini, 2018; Saturnino et al., 2012; Silva et al., 2017).

De acordo com Cruz et al. (2020), o farmacêutico precisou assumir um novo perfil devido ao processo de transição demográfica e epidemiológica, caracterizado pela inversão de aumento nas taxas de DCNT quando comparada com as doenças transmissíveis, e aumento da morbimortalidade. A partir de então, o cuidado farmacêutico começa a ser inserido como prática farmacêutica na atenção à saúde (Cruz et al., 2020).

Ainda segundo Cruz et al. (2020), o cuidado farmacêutico se traduz como uma atuação profissional do farmacêutico, na qual o mesmo assume a responsabilidade pela farmacoterapia do paciente, com o objetivo de alcançar resultados mensuráveis e traçados, de acordo com a prescrição médica, a fim de promover a qualidade de vida.

Para Naqvi et al. (2019), o cuidado farmacêutico é um serviço de saúde, com um olhar centrado no paciente, prestado por farmacêuticos, e que incorpora, mas não é limitado, a diferentes tipos de intervenções, como a gestão da terapia, autocuidado e autogestão de doenças, terapia e orientação motivacional, e educação em saúde.

Conforme Araujo et al. (2017) e Bastos-barbosa et al. (2012), o cuidado farmacêutico pode ser realizado a partir de diferentes intervenções para incrementar a adesão ao tratamento, como a prestação de orientação e sensibilização do paciente e familiares sobre a doença, a importância e os benefícios do tratamento, as particularidades da terapêutica, bem como as reações que podem surgir em decorrência da utilização de medicamentos e como manejá-las.

Silva et al. (2017), em estudo, evidenciaram que o cuidado farmacêutico direcionado para o incremento da adesão pode ser realizado a partir da orientação do uso correto de medicamentos e acompanhamento das reações adversas e interações medicamentosas, com a diminuição do surgimento de erros e a descontinuidade do tratamento.

Mary et al. (2018), demonstraram que o cuidado farmacêutico pode ser eficaz sobre a adesão a terapêutica, a partir do envio de mensagens de texto curtas para os pacientes. Já Lavielle et al. (2018), relatam que a atuação do farmacêutico sobre o gerenciamento de medicamentos é capaz de promover uma melhora na adesão ao tratamento em pacientes com DCNT.

Stockl et al. (2010), relataram melhora na adesão de pacientes que receberam acompanhamento farmacêutico realizado por telefone, como forma da prática do cuidado farmacêutico. Já Milosavljevic et al. (2018), relataram a promoção da educação em saúde e do aconselhamento ao paciente como foco da prática do farmacêutico no cuidado farmacêutico, para melhorar a aderência dos pacientes ao tratamento, a partir da racionalização da terapia medicamentosa.

Tritany e Tritany (2020), em estudo, descreve a revisão da farmacoterapia como uma das intervenções farmacêuticas, realizadas durante o cuidado farmacêutico, que evitou a permanência de erros nas prescrições e erros de medicação, e promoveu a garantia de segurança do paciente e a adesão ao tratamento.

Segundo Song et al. (2021), o acompanhamento farmacoterapêutico compreende uma das formas de atuação do 
profissional farmacêutico, que faz parte do cuidado farmacêutico, e é essencial no processo de manutenção da efetividade do tratamento e eficácia do medicamento, na garantia da segurança do paciente, e na adesão ao tratamento medicamentoso.

Li et al. (2021), descrevem o acompanhamento farmacoterapêutico, realizado pelo farmacêutico, a partir do acompanhamento de pacientes com DCNT, através da verificação, garantia e manutenção da adesão ao tratamento medicamentoso, da efetividade dos medicamentos em uso e da vigilância com relação ao surgimento de reações adversas.

As intervenções farmacêuticas, direcionadas para a melhora da adesão ao tratamento medicamentoso, podem ser executadas a partir de diferentes estratégias, e em diferentes níveis e setores da atenção à saúde, através do cuidado farmacêutico.

\section{Conclusão}

O estudo permite inferir que a adesão é um componente essencial para realização do tratamento de qualquer condição clínica, e a sua ausência é responsável pela prevalência de muitas doenças, e está associada com a piora no panorama e no quadro geral do paciente. Além de complicações no âmbito fisiopatológico, a não aderência ao tratamento medicamentoso também tem sua parcela de influência negativa no setor econômico.

A garantia e a manutenção da adesão ao tratamento é um dos objetivos que perpassa diferentes áreas da atenção à saúde. Neste contexto, o trabalho demonstrou que o farmacêutico está inserido como um dos profissionais que atuam na remoção de barreiras para adesão, a partir do cuidado farmacêutico. Visto que, tal prática farmacêutica proporciona a aproximação entre o farmacêutico e o paciente, que passa a ser o foco de atuação do profissional.

O estudo evidenciou que o cuidado farmacêutico é aplicado e utilizado como ferramenta que incrementa a adesão ao tratamento medicamentoso, em diferentes condições e problemas de saúde, principalmente para as doenças crônicas, através de diferentes tipos de intervenções, realizadas de forma individual ou coletiva, e em diferentes áreas de atuação do profissional farmacêutico.

Portanto, é possível concluir que o cuidado farmacêutico se mostra como uma prática estratégica e de extrema importância para promoção da adesão medicamentosa. A partir deste trabalho é possível fornecer achados que consolidam e estimulam a realização do cuidado farmacêutico, como prática de atuação, para melhorar a aderência ao tratamento medicamentoso. A valorização e compreensão da multifatorialidade, que compõe o processo de adesão, é algo que deve ser explorado pelo farmacêutico, em trabalhos futuros, para realização de um serviço consciente, de acordo com a realidade de cada indivíduo.

\section{Referências}

Agostini, C. P. (2018). Cuidado farmacêutico no Brasil: uma revisão bibliográfica. [Trabalho de conclusão de curso, Universidade Regional Integrada do Alto Uruguai e das Missões]. http://repositorio.uricer.edu.br/handle/35974/180

Araujo, N. C. F., Palhão, D. M. R., Silva, V. C., Ávila, J. O. L., Cardoso, K. F., Santos, E. R. F., Lomba, F. C. M. S., Carvalho, I. R. A., Souza, B. Q., \& Polisel, C.G. (2017). Avaliação da adesão ao tratamento em condições crônicas de saúde por meio do cuidado farmacêutico. Revista Brasileira de Farmácia Hospitalar e Serviços de saúde, 08 (03), 37-41. http://rbfhss.saude.ws/revista/arquivos/2017080306001194BR.pdf

Azevedo, M. G. B., Pedrosa, R. S., Aoqui, C. M., Martins, R. R., \& Junior, T. N. (2017). Effectiveness of home pharmaceutical interventions in metabolic syndrome: a randomized controlled trial. Brazilian Journal of Pharmaceutical Sciences, 53 (02), 01-09. https://doi.org/10.1590/s2175-97902017000216089

Bastos-barbosa, R. G., Ferriolli, E., Moriguti, J. C., Nogueira, C. B., Nobre, F., Ueta, J., \& Lima, N. K.C. (2012). Adesão ao tratamento e controle da pressão arterial em idosos com hipertensão. Arquivos Brasileiros de Cardiologia, 99 (01), 636-641. https://doi.org/10.1590/S0066-782X2012005000054

Chambela, M. C., Mediano, M. F. F., Carneiro, F. M., Ferreira, R. R., Waghabi, M. C., Mendes, V. G., Oliveira, L.S., Holanda, M.T., Souza, A. S., Costa, A. R., Xavier, S. S., Silva, G. M. S., \& Saraiva, R. M. (2019). Impact of pharmaceutical care on the quality of life of patients with heart failure due to chronic Chagas disease: Randomized clinical trial. British Journal of Clinical Pharmacology, 01 (86), 143-154. https://doi.org/10.1111/bcp.14152

Chung, W. W., Chua, S. S., Lai, P. S. M., \& Chan, S. P. (2014). Effects of a pharmaceutical care model on medication adherence and glycemic control of 
Research, Society and Development, v. 10, n. 16, e316101623753, 2021 (CC BY 4.0) | ISSN 2525-3409 | DOI: http://dx.doi.org/10.33448/rsd-v10i16.23753

people with type 2 diabetes. Patient Preference and Adherence, 03 (08), 85-94. https://doi.org/10.2147/PPA.S66619

Cruz, W. M., Queiroz, L. M. D., \& Soler, O. (2020). Cuidado farmacêutico para utentes de farmácia comunitária privada: Revisão sistemática. Brazilian Journal of Development, 06 (10), 78682-78702. https://www.brazilianjournals.com/index.php/BRJD/article/view/18343

Gerenutti, M., Martinez, A. M. V., \& Bergamaschi, C. C. (2017). The effectiveness of a pharmaceutical care model on adherence to antiretroviral therapy: as AME-based cohort study in brazil. Advanced Pharmaceutical Bulletin, 07 (03), 469-472. https://www.ncbi.nlm.nih.gov/pmc/articles/PMC5651069/

Gewehr, D. M., Bandeira, V. A. C., Gelatti, G. T., Colet, C. F., \& Oliveira, K. R. (2018). Adesão ao tratamento farmacológico da hipertensão arterial na Atenção Primária à Saúde. Saúde Debate, 42 (116), 179-190. https://www.scielo.br/j/sdeb/a/4Dh4vDYyPWvKHSxHzT9X7zf/?lang=pt\&format=pdf

Haramiova, Z., Stasko, M., Hulin, M., Tesar, T., Kuzelova, M., \& Morisky, D. M. (2017). The effectiveness of daily SMS reminders in pharmaceutical care of older adults on improving patients' adherence to antihypertensive medication (SPPA): study protocol for a randomized controlled trial. BioMed Central, 18 (334), 01-15. https://trialsjournal.biomedcentral.com/track/pdf/10.1186/s13063-017-2063-8.pdf

Heisler, M., Hofer, T. P., Schmittdiel, J. A., Selby, J.V., Klamerus, M. L., Bosworth, H. B., Bermann, M., \& Kerr, E. A. (2012). Improving blood pressure control through a clinical pharmacist outreach program in patients with diabetes mellitus in 2 high-performing health systems: the adher-ence and intensification of medications cluster randomized, controlled pragmatic trial. Circulation, 125 (23), 2863-2872. https://www.ncbi.nlm.nih.gov/pmc/articles/PMC3999872/

Hernández, C. M. L., Rodríguez, J. A. L., Fernández, F. L., Larrañaga, A. C., Cortes, J. B., Feliu, L. A. G., Plou, B. P., \& González, I. D. C. (2020). Social support, social context and nonadherence to treatment in young senior patients with multimorbidity and polypharmacy followed-up in primary care. Plos One, 15 (06), 01-15. https://www.ncbi.nlm.nih.gov/pmc/articles/PMC7314051/

Ho, M. P., Bryson, C. L., \& Rumsfeld, J. S. (2009). Medication adherence: its importance in cardiovascular outcomes. Circulation, 119 (23), $3028-3035$. https://pubmed.ncbi.nlm.nih.gov/19528344/

Holt, E. W., Muntner, P, Joyce, C. J., Webber, L., \& Wood, M. (2010). Health-related quality of life and antihypertensive medication adherence among older adults. Age Ageing, 39 (04), 481-487. https://doi.org/10.1093/ageing/afq040

Khdour, M. R., Elyan, S. O., Hallak, H. O., Jarab, A. S., Mukattash, T. L., \& Astal, A. (2019). Pharmaceutical care for adult asthma patients: A controlled intervention one-year follow-up study. Basic \& Clinal Pharmacology \& Toxicology, 01 (126), 332-340. https://doi.org/10.1111/bcpt.13344

Lavielle, M., Puyraimond-zemmour, D., Romand, X., Gossec, L., Senbel, E., Pouplin, S., Beauvais, C., Gutermann, L., Mezieres, M., Dougados, M., \& Molto, A. (2018). Methods to improve medication adherence in patients with chronic inflammatory rheumatic diseases: a systematic literature. Rheumatic and Musculoskeletal diseases, 04 (02), 01-08. http://dx.doi.org/10.1136/rmdopen-2018-000684

Li, H., Zheng, S., Liu, F., \& Zhao, R. (2021). Fighting against COVID-19: Innovative strategies for clinical pharmacists. Research in Social and Administrative Pharmacy, 01 (17), 1813-1818. https://doi.org/10.1016/j.sapharm.2020.04.003

Lu, M., Zhou, Y., Wang, B., Hu, Z., Du, Y., Liu, S., Lin, X., Cui, Y., \& Jin, H. (2018). Impact of multidisciplinary collaborative pharmaceutical care on knowledge, adherence, and efficacy of hormone therapy in climacteric women. Patient Preference and Adherence, 01 (12), 1273-1278. https://doi.org/10.2147/PPA.S165238

Marfo, A. F. A., \& Daaku, F. T. O. (2017). Exploring the extended role of the community pharmacist in improving blood pressure control among hypertensive patients in a developing setting. Journal of Pharmaceutical Policy and Practice, 10 (39), 01-09. https://www.ncbi.nlm.nih.gov/pmc/articles/PMC5740879/

Mary, A., Boursier, A., Henry, I. D., Grados, F., Séjourné, A., Salomon, S., Fardellone, P., Brazier, M., \& Goeb, V. (2018). Mobile phone text messages improve treatment adherence in patients taking methotrexate for rheumatoid arthritis: a randomized pilot study. Arthritis Care \& Research, 01 (01), 01-26. https://doi.org/10.1002/acr.23750

Martin, D., Luz, P.M., Lake, J. E., Clark, J. L., Campos, D. P., Veloso, V. G., Moreira, R. I., Cardoso, S. W., Klausner, J. D., \& Grinsztejn, B. (2017). Pharmacy refill data can be used to predict virologic failure for patients on antiretroviral therapy in Brazil.Journal of the International AIDS Society, 20 (01), 01-05. https://www.arca.fiocruz.br/handle/icict/25689

Menditto, E., Orlando, V., Rosa, G., Minghetti, P., Musazzi, U. M., Cahir, C., Kurczewska-Michalak, M., Kardas, P., Costa, E., Lobo, J. M. S., \& Almeida, I. F. (2020). Patient centric pharmaceutical drug product design - the impact on medication adherence. Pharmaceutics, 44 (12), 01-33. https://www.ncbi.nlm.nih.gov/pmc/articles/PMC7023035/

Milosavljevic, A., Aspden, T., \& Harrison, Jeff. (2018). Community pharmacist-led interventions and their impact on patients' medication adherence and other health outcomes: a systematic review. International Journal of Pharmacy Practice, 01 (26), 387-397. https://doi.org/10.1111/ijpp.12462

Modé, C. L., Lima, M. M., Carnavalli, F., Trindade, A. B., Almeida, A. E., Chin, C. M., \& Santos, J. L. (2015). Atenção farmacêutica em pacientes hipertensos: estudo piloto. Revista de Ciências Farmacêuticas Básica e Aplicada, 36 (01), 35-41. https://rcfba.fcfar.unesp.br/index.php/ojs/article/view/65/63

Naqvi, A. A., Hassali, M.A., Naqvi, S. B. S., \& Aftab, M. T. (2019). Impact of pharmacist educational intervention on disease knowledge,rehabilitation and medication adherence,treatment-induced direct cost, health related quality of life and satisfaction inpatients with rheumatoid arthritis: study protocol for a randomized controlled trial. BMC research in progress, 20 (488),01-11. https://pubmed.ncbi.nlm.nih.gov/31399128/

Nicoletti, M., A. \& Ito, R. K. (2018). Formação do farmacêutico: novo cenário de atuação profissional com o empoderamento de atribuições clínicas. Revista Saúde, 11 (03-04), 01-14. http://revistas.ung.br/index.php/saude/article/view/2536/2395

Oliveira, R. E. M., Filipin, M. D. V., \& Giardini, M. H. (2015). Intervenções farmacêuticas destinadas à otimização da adesão ao tratamento medicamentoso de um paciente. Revista Eletrônica de Farmácia, 12 (02), 39-51. https://doi.org/10.5216/ref.v12i2.34346 
Research, Society and Development, v. 10, n. 16, e316101623753, 2021 (CC BY 4.0) | ISSN 2525-3409 | DOI: http://dx.doi.org/10.33448/rsd-v10i16.23753

Plaster, C. P., Melo, D. T., Boldt, V., Cassaro, K. O. S., Lessa, F.C. R., Boechat, G. A. P., Bissoli, N. S., \& Andarade, T. U. (2012). Reduction of cardiovascular risk in patients with metabolic syndrome in a community health center after a pharmaceutical care program of pharmacotherapy follow-up. Brazilian Journal of Pharmaceutical Sciences, 48 (03), 435-446. https://www.scielo.br/j/bjps/a/KRHFrqQ5hrHh7q4Lfw6LYVy/?format=pdf\&lang=en

Rigoni, C. C., Brito, E. S., Alano, G. M., \& Galato, D. (2015). Pharmacotherapy review: a proposal to improve medication adherence among hypertensive patients. Brazilian Journal of Pharmaceutical Sciences, 51 (04), 763-773. https://doi.org/10.1590/S1984-82502015000400002

Saleem, F., Hassali, M. A., Shafie, A. A., Haq, N. U., Faraooqui, M., Aljadhay, H., \& Ahmad, F. U. D. (2013). Pharmacist intervention in improving hypertension related knowledge, treatment medication adherence and health-related quality of life: a non-clinical randomized controlled trial. Health Expectations, 01 (18), 1270-1281. https://doi.org/10.1111/hex.12101

Saturnino, L. T. M., Perine, E., Luz, Z. M. P., \& Modena, C. M. (2012). Farmacêutico: um profissional em busca de sua identidade. Revista Brasileira de Farmácia, 93 (01),10-16. https://www.arca.fiocruz.br/handle/icict/7860

Silva, L. C. A., Brito, P. O. L., Melo, C. D., Falcai, A., \& Pereira, I. C.P. (2017). Contribuições da atenção farmacêutica a pacientes em tratamento oncológico. Revista de Investigação Biomédica, 09 (02), 216-222. http://www.ceuma.br/portalderevistas/index.php/RIB/article/view/164/pdf

Soares, A. L. P. P. P., Costa, M. A., \& Teixeira, J. J. V. (2016). Nível de entendimento sobre prescrição farmacêutica. Estamos preparados para essa nova realidade? Infarma Ciências Farmacêuticas, 28 (03), 149-156. http://dx.doi.org/10.14450/2318-9312.v28.e3.a2016.pp149-156

Song, Z., Hu, Y., Zheng, S., Yang, L., \& Zhao, R. (2021). Hospital pharmacists' pharmaceutical care for hospitalized patients with COVID-19: Recommendations and guidance from clinical experience. Research in Social and Administrative Pharmacy, 01 (17), 2027-2031. https://www.ncbi.nlm.nih.gov/pmc/articles/PMC7129111/

Stockl, K. M., Shin, J. S., Lew, H. C., Zakharyan, A., Harada, A. S. M., Solow, B. K., \& Curtis, B. S. (2010). Outcomes of a rheumatoid arthritis disease therapy management program focusing on medication adherence. Journal of Managed Care \& Specialty Pharmacy, 16 (08), 593-604. https://doi.org/10.18553/jmcp.2010.16.8.593

Tommelein, E., Mehuys, E., Van Hees, T., Adriaens, E., Van Bortel, L., Christiaens, T., Van Tongelen, I., Remon, J. P., Boussery, k., \& Brusselleet, G. (2013). Effectiveness of pharmaceutical care for patients with chronic obstructive pulmonar disease (PHARMACOP): a randomized controlled trial. British Journal of Clinical Pharmacology, 77 (05), 756-766. https://doi.org/10.1111/bcp.12242

Tritany, R. F., \& Tritany, E. F. (2020). Serviços Farmacêuticos no Enfrentamento à COVID-19: Uma Revisão Integrativa da Literatura. Revista Saúde em Redes, 06 (02), 07-24. http://revista.redeunida.org.br/ojs/index.php/rede-unida/article/viewFile/3301/536

Organização Mundial da Saúde. (2003). Adherence to Long-term Therapies: Evidence for Action. https://apps.who.int/iris/handle/10665/42682

Wuyts, J., Maesschalck., J., Wulf, I., Foubert, K., Boussery, K., Lepeleire, J., \& Foulon, V. (2018). Studying the impact of a medication use evaluation for polymedicated older patients by the community pharmacist (SIMENON):study protocol. BMC Health Services Research, 18, (623), 01-08. https://bmchealthservres.biomedcentral.com/articles/10.1186/s12913-018-3440-Z

Xin, C., Xia, Z., Jiang, C., Lin, M., \& Li, G. (2015). Effect of pharmaceutical care on medication adherence of patients newly prescribed insulin therapy: a randomized controlled study. Patient Preference and Adherence, 01 (09), 797-802. https://doi.org/10.2147/PPA.S84411 